\title{
Queens of the inquiline social parasite Acromyrmex insinuator can join nest-founding queens of its host, the leaf-cutting ant Acromyrmex echinatior
}

\author{
J. Howe $\mathrm{e}^{1,2} \cdot$ M. Schiøtt ${ }^{1,3}$ (D) J. J. Boomsma ${ }^{1}(\mathbb{D}$
}

Received: 17 December 2020 / Revised: 29 March 2021 / Accepted: 26 May 2021 / Published online: 17 June 2021

(c) The Author(s) 2021

\begin{abstract}
Queens of the inquiline social parasite Acromyrmex insinuator are known to infiltrate mature colonies of Acromyrmex echinatior and to exploit the host's perennial workforce by producing predominantly reproductive individuals while suppressing host reproduction. Here we report three cases of an A. insinuator queen having joined an incipient colony of A. echinatior that contained only the founding host-queen and her small symbiotic fungus garden. We conjectured that 1:1 host-inquiline co-founding - a phenomenon that has only rarely been reported in ants-may imply that the presence of an A. insinuator queen may incur benefits to the host by increasing survival of its incipient colonies. We observed that the parasite queens neither foraged nor defended the nest against intruders. However, the parasite queens interacted with the host and fungus in a way that could be consistent with grooming and/or with contributing eggs. These observations may help explain why $A$. insinuator queens have maintained metapleural glands, even though they are smaller than those of host queens, and why $A$. insinuator has lost the large foraging worker caste but not the small worker caste.
\end{abstract}

Keywords Parasitism $\cdot$ Mutualism $\cdot$ Inquiline

Acromyrmex insinuator is a queen-tolerant inquiline that parasitizes its sister species Acromyrmex echinatior, a leafcutting ant native to Panama (Schultz et al. 1998; Sumner et al. 2004). It infiltrates mature A. echinatior colonies where, as in other inquilines, it produces predominantly reproductive offspring. However, the species is unusual as it has lost the large worker caste but still produces small workers (Sumner et al. 2003a, c, 2004; Buschninger 2009; Schrader et al. 2021). As a relatively recently evolved parasite, A. insinuator is thought to represent a somewhat transitional state: it still closely resembles its host species, although it is slightly miniaturised, and its minor workers

J. Howe

jack.howe@zoo.ox.ac.uk

1 Centre for Social Evolution, Department of Biology, University of Copenhagen, Universitetsparken 15, 2100 Copenhagen, Denmark

2 Present Address: Department of Zoology, University of Oxford, Oxford, UK

3 Present Address: National Institute of Aquatic Resources, Technical University of Denmark, 2800 Kongens Lyngby, Denmark may be necessary for suppression of host reproduction or early nursing of inquiline brood (Sumner et al. 2003a). Infection by A. insinuator is ultimately fatal for host colonies in the lab, although it may take years until parasite queens (often present in multiples) suppress host queen oviposition so large foraging workers are no longer replaced. This appears to give the parasites only a single season to produce a semelparous clutch of male and female reproductives which exhausts the colony's symbiotic fungus garden and makes the colony collapse by the time the inquiline's winged reproductives disperse (Bekkevold and Boomsma 2001). It is possible, however, that parasite queens may co-reproduce with the host queen for several years in large field colonies when host colonies thrive and come to maintain many fungus gardens (Bekkevold and Boomsma 2001).

During a recent field experiment that involved following newly-founded Acromyrmex nests in and around Gamboa, Panama (Howe et al. 2019), we discovered an additional mechanism by which inquilines and hosts associate. In three successive years (2015-2017), shortly after the Acromyrmex mating flight in April/May, we thoroughly surveyed a total of nine plots across six sites for newlyfounded Acromyrmex echinatior and A. octospinosus nests. 
Four of the six sites lay within Gamboa, while the remaining two lay approximately $6 \mathrm{~km}$ south-east. At this stage of incipient colony founding, nests contain only a recently inseminated queen and a fungus garden of a diameter similar to her body length and, while brood is usually present, the first workers have yet to hatch; see Howe et al. (2019) for more detailed information. That study identified a total of 90 incipient A. echinatior nests and 73 A. octospinosus nests and in the third year, three of the 56 nests identified at one site, Lorenzo Bank, contained an additional queen of Acromyrmex insinuator - in each case with an A. echinatior host queen, the primary host of $A$. insinuator. This possibility of host-inquiline co-founding was suggested as a possibility by Schultz et al. (1998) but has, to our knowledge, only been occasionally recorded in Megalomyrmex species that exploit other attine ants (Boudinot et al. 2013) and in Tetramorium inquilinum (formerly Teleutomyrmex schneideri) whose miniature queens hitchhike on the back of host queens-to-be during dispersal flights (Hölldobler and Wilson 1990).

This triple observation made us consider the possible costs and benefits of this unexpected type of association. We conjectured that queen mortality in the nest founding stage is extremely high, so that founding host queens-which otherwise almost always appear to be lone haplometrotic foundresses in A. echinatior; cf. Nehring et al. (2018)—could potentially incur a competitive advantage from the presence of an extra cofounding queen in terms of defence against intruders or pathogens. If that were the case, it would imply that the interaction may be mutualistic rather than parasitic at the colony founding stage, and that early host-colony survivorship could more than compensate for the loss of reproductive success to the parasite towards the end of the colony cycle.

A. echinatior queens found colonies non-claustrally. Before the first workers emerge, they regularly leave the nest to forage for plant material to nourish their incipient fungal garden (Fernandez-Marin et al. 2004). This has several costs: the host queen is vulnerable to predation while foraging, she is potentially exposed to diseases, and her fungus is left unguarded during each foraging trip and at risk of being stolen by a neighbouring queen (Howe et al. 2019) or by fungivorous insects. The presence of an A. insinuator queen may thus provide tangible benefits to the host in the form of hygienic services if the parasite queen were to groom the host queen, or if she were to function as an additional guard or additional egg-layer to boost the size of the first worker brood. The question then becomes whether this early-life enhanced likelihood of eventually producing a mature colony large enough to reproduce (i.e. a fungus garden volume of at least $1 \mathrm{~L}$ ) is substantial enough to compensate for later years of lost reproduction. In other words, would an average founding queen with an inquiline companion be better or worse off than an average haplometrotic queen founding a colony on her own?

From a parasite queen's perspective, a recently-founded nest that is routinely unguarded while the host queen is foraging would likely be easier to infiltrate than a mature colony with an aggressively defensive workforce (Larsen et al. 2014). Infiltrating a young colony could thus increase her short-term likelihood of survival, although her odds of perishing with the incipient colony would be severe relative to a more stable mature host colony, and it would take more years on average to possibly realise any reproductive success. Thus, although the host and parasite will always be in conflict over mutually exclusive reproduction later in their joint colony's life cycle, in these early stages, they may both stand to gain from 'parasitism' during colony founding and will have aligned interests in ensuring colony growth and survival until it reaches a sufficient size to reproduce. The question is, therefore, whether three cases of observed cooccurrence of founding queens represents a strategy of at least one and possibly both species, or whether they remain merely haphazard coincidences.

To test these speculations, each of the three incipient nests with a paired host and parasite queen was carefully excavated and housed in a $6 \mathrm{~cm}$ wide Petri-dish to contain the fungus garden opening via a small hole on the side into a $15 \mathrm{~cm}$ wide Petri-dish that functioned as foraging arena. Colonies were maintained at ambient temperature in Panama, and were provided daily with fresh leaves and moist cotton wool within the foraging arena. We observed the queens for a total of $10.5 \mathrm{~h}(\sim 3.5 \mathrm{~h}$ at each nest), recording foraging trips and three-way interactions between each of the queens and the fungus garden. We also confronted the three nests with conspecific intruders, by introducing individual ants-either another founding $A$. echinatior queen from the same site, or a large worker from a mature colony $(n=3$ in both cases) - into the foraging arena and, after $1 \mathrm{~min}$ acclimation, counting all behaviours from the queens directed towards the intruders for a maximum of $6 \mathrm{~min}$, with trials cut short if any party appeared to be coming to any harm. In one trial, a smaller $10 \mathrm{~cm}$ foraging arena was used, but as this did not appear to affect the behaviour of either queen, the data were pooled with the other observations. All behaviours were recorded using the software BORIS (Friard and Gamba 2016), and analysed in R (R Core Team 2013). After having been kept in Panama for 14 days, colonies were transported to Copenhagen, Denmark and maintained at ca. $25^{\circ} \mathrm{C}$ and $70 \%$ relative humidity. Unfortunately, all three nests perished within 2 weeks of relocation, so only the six preliminary trials in Panama were conducted and reported here.

There appeared to be clear differences between host and parasite queen behaviour, although the data were too few to draw robust conclusions. As expected from the fact that the inquiline queens have lost the ability to found colonies on 
their own, the host queens left the nest more often, spending more time outside of the nest (Fig. 1A), although no recorded excursions resulted in material being foraged. Only one parasite queen made a brief excursion out of the nest (Fig. 1A, B), and none of the three parasite queens were observed foraging or manipulating foraged materialalthough the differences in the number of excursions did not reach significance, most likely due to the paucity of data (paired Wilcoxon signed-rank test, $V=6, p=0.25$ ). Host and parasite queens spent a similar proportion of time in contact with the fungal symbiont (Fig. 1A) and both appeared to work the fungus-garden in a similar manner. We often observed interactions between the paired queens (Fig. 1C), with both parasite and host antennating and apparently grooming each other. These interactions were often initiated by the parasite, potentially consistent with inquilines offering hygienic service to the hosts rather than vice versa. The aggression trials (Fig. 2) showed clear behavioural differences with the host-queen doing essentially all pursuit and fighting, and inquiline queens at most antennating and only

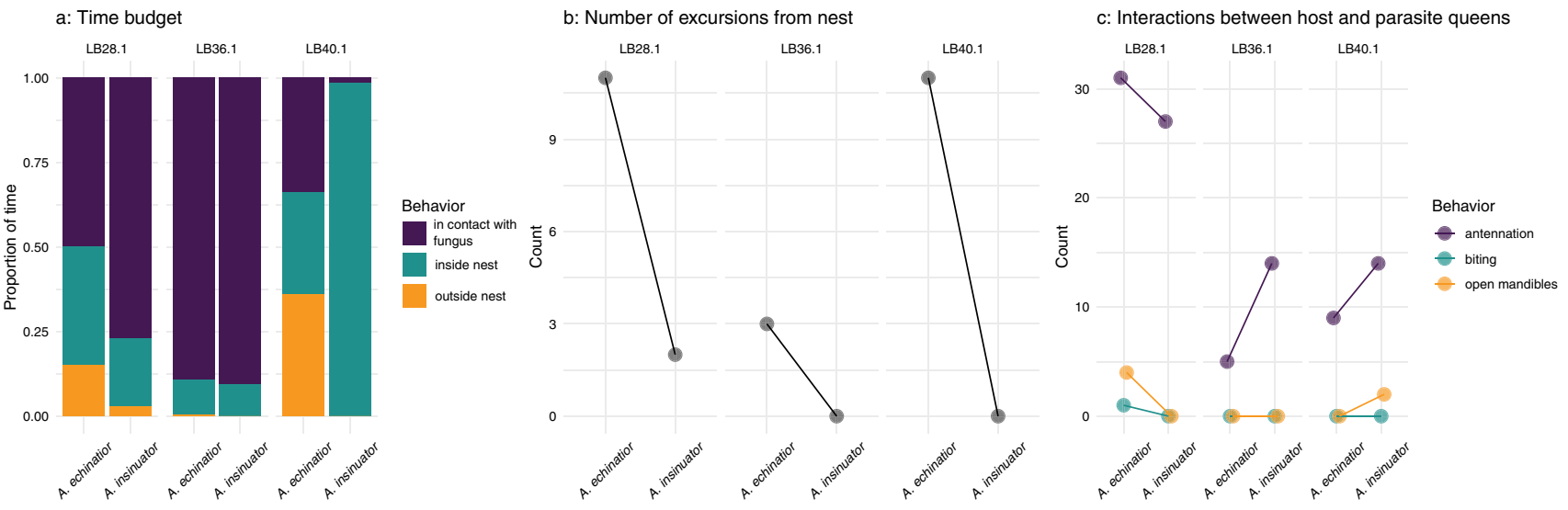

Fig. 1 Behavioural observations on natural pairs of queens of Acromyrmex echinatior hosts and Acromyrmex insinuator inquiline parasites. a Although results varied across colonies partly because of factors beyond our control (e.g. nest 2 showing almost no foraging at all), there was a clear tendency for host queens to do all of the foraging outside of the inner petri dish containing the garden (orange). When inside the nest, host and parasite queens spent a similar amount of time in contact with the fungus garden in two colonies (blue), but not in the third where there was almost no fungus left. b The greater proportion of time spent outside of the nest is reflected in the greater number of excursions taken by the A. echinatior host queens in all nests, although none of the observed excursions resulted in material being collected. c Host and parasite queens interacted with high frequency without obvious signs of aggression, but antennation and behaviours that appear consistent with grooming were regularly observed. Some rare, very mild forms of potential aggression (opening mandibles) were observed in two of three nests, but they rapidly dissipated and did not appear to preclude or interrupt friendly interactions. Labels above each figure column refer to the three nests housed in separate Petri dishes

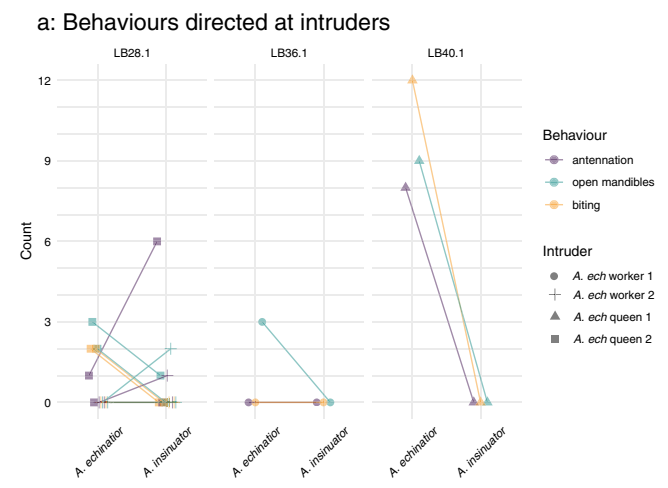

b: Typical differences in aggression

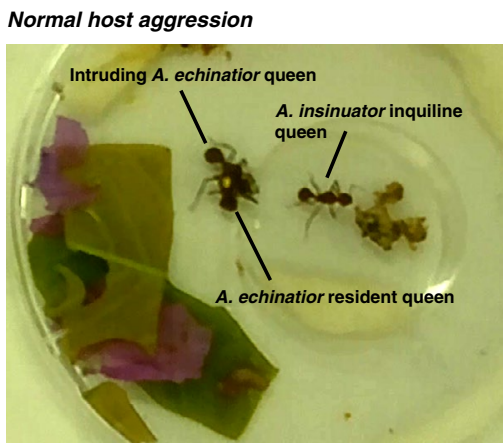

Lack of parasite aggression

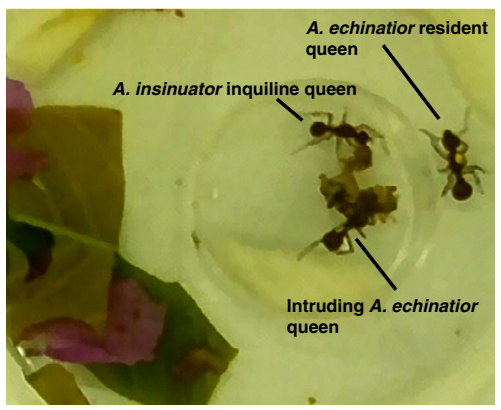

Fig. 2 Hosts and inquiline parasites differ in aggressive defence against intruders. a Host queens were more aggressive than parasite queens, with results pooled across intruder types-host queens would aggressively chase and bite intruders while parasite queens generally appeared to ignore them. Labels above each figure column refer to the three nests housed in separate Petri dishes. b Stills were taken from videos of behavioural trials showing typical reactions of host and parasite queens to intruders, with host queens attacking intruders and parasite queens refraining from aggression even when the host queen was not in the nest. Stills have been sharpened, and the exposure increased using Adobe Photoshop 
very occasionally opening her mandibles. Although intruding queens appeared to be repelled more aggressively than intruding workers, the host queen appeared more aggressive than the parasite queen regardless of intruder identity, so we pooled these results to increase the limited statistical power. While the two queens showed no significant difference in non-aggressive antennation directed towards the intruder (paired Wilcoxon signed-rank test, $V=3, p=1$ ), the A. echinatior host displayed significantly more aggressive posturing of the mandibles and biting than the A. insinuator parasite (paired Wilcoxon signed-rank test, $V=33, p=0.037$ ). This seems inconsistent with a putative nest guarding role by A. insinuator queens pairing up with a host queen during colony founding.

Our results imply that there are two potential explanations for the occasional presence of parasitic $A$. insinuator queens in incipient $A$. echinatior nests. First, infiltrating a recently founded host nest may simply be maladaptive because young host colonies are too small to support parasite reproduction and none of these paired colonies would therefore have survived beyond the founding stage. Perhaps these $A$. insinuator queens could not find a mature A. echinatior nest after their nuptial flight, so settling into an incipient nest was the only alternative. Over the 3 years in which we monitored founding colonies at this site, we have not observed a single mature Acromyrmex colony nearby, which would be consistent with this null hypothesis. This null hypothesis is also consistent with none of the colonies surviving long in the lab. However, the survival probability of a parasitised nest did not appear to differ significantly from unparasitised nests (exact binomial test, $p=1$ ): only 11 of 100 lone Acromyrmex queens collected that year were reared to mature lab colonies, with none surviving from that specific site. Under this scenario, the parasites might then simply have expressed the behaviours that are adaptive when parasitising mature colonies. That the parasite spent so much time in contact with and handling the fungus could therefore be an attempt to acquire the colony's odour as insinuating queens need to do in a mature host colony (Sumner et al. 2003c), and inter-queen interactions could be interpreted as the parasite appeasing the host queen in an attempt to avoid aggression. Likewise, the lack of aggression towards intruders might reflect normal parasite queen behaviour because there are usually hundreds if not thousands of large workers in a mature host colony that will aggressively defend the colony. The hosts' multiple excursions without collecting useful material could, in this context, be interpreted as attempts to find another unparasitised nest or vacated fungus garden: founding Acromyrmex queens regularly usurp the nests of neighbours (Howe et al. 2019). Our artificial set-up did not allow us to test this, however. Further, this scenario requires that the host queens are 'aware' that their nest is parasitised, and one might expect them to simply evict the smaller parasite queen rather than undergo a costly move.

Alternatively, the paired arrangements we observed could represent an alternative to the default parasite strategy of insinuating on a mature host colony. A recently founded nest is often unguarded because the host queen needs to forage. This implies that a founding host colony is easy to invade, while the host queen may be willing to tolerate the presence of the inquiline at this stage if it would help her to gain a competitive edge over lone founding queens in neighbouring nests. We note that this potential alternative strategy is unlikely to be a genetic difference with the previously described behaviour of $A$. insinuator, which were collected in the same Gamboa area (Sumner et al. 2003a, b, 2004; Schultz et al. 1998). We found no support for the possibility that $A$. insinuator queens serve as nest guards during host foraging trips, but this does not necessarily imply that their presence does not act as a deterrent when the host queen is absent. Previous observations suggest that queens that have lost their incipient garden are more likely to steal from unoccupied incipient nests in the close vicinity (Howe et al. 2019), so the presence of an A. insinuator queen may deter stealing and thus confer a benefit without necessarily resorting to overt aggression. Likewise, we cannot rule out potential hygienic benefits because the A. insinuator queens interacted with both the host-queen and the fungus garden (Figs. 1,2). As we did not assess the eggs present in the nest, we cannot refute a potential role as a second egglayer that contributes to more rapidly establishing a larger worker cohort, or trophic-eggs contributions that increase host-queen productivity. If this adaptive hypothesis is correct, it could potentially explain two unusual characteristics of $A$. insinuator as an inquiline social parasite: (i) that $A$. insinuator queens still invest in metapleural glands (which are reduced in A. insinuator workers) (Sumner et al. 2003a), and (ii) that $A$. insinuator has not completely lost its worker castes, as older and evolutionarily more derived Acromyrmex inquilines have (Rabeling et al. 2015; Hölldobler and Wilson 1990; Gallardo 1929; Schrader et al. 2021). A. insinuator queens rarely produce any larger (foraging) workers, but they always produce small workers that are thought to be important in raising parasite brood and for ultimately suppressing fertility of the host queen (Sumner et al. 2003a). We were unable to test whether $A$. insinuator queens would have defended the fungus garden in case an intruder intent on stealing the incipient garden arrived while the founding host queen was away, although this scenario did occur once during the aggression trials and the resident $A$. insinuator queen did not defend the nest (Fig. 2B).

Although the inquiline queens in founding colonies did not show any behaviours that have not been observed when they parasitise mature colonies, we cannot yet rule out a potential benefit to hosting an $A$. insinuator inquiline 
early during colony development. As long as that ambiguity persists, the possibility for an adaptive explanation is of considerable interest, because no inquiline ant has yet been suggested to possibly have beneficial effects before it exploits the host. We note, however, that a case of a completely unrelated guest ant conferring both benefits and costs has been reported for the interaction between Megalomyrmex symmetochus guest ants and Sericomyrmex amabilis host ants in mature colonies (Adams et al. 2013), and that 1:1 co-founding has occasionally been inferred in other Megalomyrmex species interacting with fungus-growing ants, as in Megalomyrmex adamsae where the parasite queens have been observed infiltrating newly founded nests of Mycetomoellerius (formerly Trachymyr$m e x)$ zeteki that also consist of a lone queen and an incipient fungus garden (Boudinot et al. 2013; Adams et al. 2012). Megalomyrmex symmetochus guest ants impose substantial costs on Sericomyrmex host colonies in that they feed on host brood and the fungus garden and clip the wings of young gynes produced when the host colony has become mature (Adams et al. 2013). They provide benefits that more than compensate for this added burden, however, in the form of an effective defence against other fungus-raiding species (Adams et al. 2013).

In case future work would allow more pairs of founding A. echinatior-insinuator queens to be collected, we suggest that the following experiments could be done to potentially refute the adaptive hypothesis in a field plot setup reminiscent of the one used by Howe et al. (2019). First, an A. echinatior host queen in search of a replacement fungal symbiont should prefer to steal from unoccupied nests, rather than from nests containing a lone A. insinuator queen. Second, an A. echinatior host queen cohabiting with an A. insinuator queen should suffer from fewer infections, as should her fungus garden. Third, the first worker cohort to hatch in a colony with an additional $A$. insinuator queen should be larger than in non-parasitized control colonies, either through direct contribution of $A$. insinuator workers or by enhanced host queen productivity. If none of these predictions were to hold up, it would be most parsimonious to stick to the non-adaptive null-hypothesis formulated above. However, our observations add to the notion that the possible adaptive significance of life history traits in evolutionary young inquiline ants remains poorly understood, because it is a challenge to collect these rare ants and subject them to experiments (Boudinot et al. 2013; Schär and Nash 2014).

Acknowledgements We are grateful to the Smithsonian Tropical Research Institute in Panama, who provided access to their laboratory facilities, and to the Autoridad Nacional del Ambiente of Panama, who issued collection and import permits. Special thanks are extended to Aaron O'Dea and family for access to the Lorenzo Bank site in their front-yard.
Author contributions All authors contributed to the conception of this study, JH conducted collection and behavioural observations, and wrote the manuscript with input and comments from JJB and MS.

Funding This work was funded by an ERC Advanced Grant (Number 323085) awarded to JJB.

Data availability All data and code, including raw videos are available at figshare.com. For observations of nests, see https://doi.org/10.6084/ m9.figshare.14762928 and for intruder challenges, see https://doi.org/ 10.6084/m9.figshare. 14762844 .

\section{Declarations}

Conflict of interest The authors declare that they have no conflict of interest.

Open Access This article is licensed under a Creative Commons Attribution 4.0 International License, which permits use, sharing, adaptation, distribution and reproduction in any medium or format, as long as you give appropriate credit to the original author(s) and the source, provide a link to the Creative Commons licence, and indicate if changes were made. The images or other third party material in this article are included in the article's Creative Commons licence, unless indicated otherwise in a credit line to the material. If material is not included in the article's Creative Commons licence and your intended use is not permitted by statutory regulation or exceeds the permitted use, you will need to obtain permission directly from the copyright holder. To view a copy of this licence, visit http://creativecommons.org/licenses/by/4.0/.

\section{References}

Adams RMM, Shah K, Antonov LD, Mueller UG (2012) Fitness consequences of nest infiltration by the mutualist-exploiter Megalomyrmex Adamsae. Ecol Entomol 37:453-462

Adams RM, Liberti J, Illum AA, Jones TH, Nash DR, Boomsma JJ (2013) Chemically armed mercenary ants protect fungus-farming societies. Proc Natl Acad Sci USA 110:15752-15757

Bekkevold N, Boomsma N (2001) Evolutionary transition to a semelparous life history in the socially parasitic ant Acromyrmex insinuator. J Evol Biol 13:615-623

Boudinot BE, Sumnicht TP, Adams RM (2013) Central American ants of the genus Megalomyrmex Forel (Hymenoptera: Formicidae): six new species and keys to workers and males. Zootaxa 3732:1-82

Buschninger A (2009) Social parasitism among ants: a review. Myrmecol News 12:219-235

Fernandez-Marin H, Zimmerman JK, Wcislo WT (2004) Ecological traits and evolutionary sequence of nest establishment in fungusgrowing ants (Hymenoptera, Formicidae, Attini). Biol J Linn Soc $81: 39-48$

Friard O, Gamba M (2016) BORIS: a free, versatile open-source eventlogging software for video/audio coding and live observations. Methods Ecol Evol 7:1325-1330

Gallardo A (1929) Note sur les moeurs de la fourmi Pseudoatta Argentina. Revista De La Sociedad Entomológica Argentina 10:197-202

Hölldobler B, Wilson EO (1990) The ants. Harvard University Press

Howe J, Schiøtt M, Boomsma JJ (2019) Horizontal partner exchange does not preclude stable mutualism in fungus-growing ants. Behav Ecol 30:372-382 
Larsen J, Fouks B, Bos N, D'ettorre P, Nehring V (2014) Variation in nestmate recognition ability among polymorphic leaf-cutting ant workers. J Insect Physiol 70:59-66

Nehring V, Dijkstra M, Sumner S, Hughes W, Boosmsma K (2018) Reconstructing the relatedness of cooperatively breeding queens in the panamanian leaf-cutting ant Acromyrmex echinatior (Hymenoptera: Formicidae). Myrmecol News 27:33-45

R Core Team (2020) R: A language and environment for statistical computing. R Foundation for Statistical Computing, Vienna, Austria. https://www.R-project.org/

Rabeling C, Schultz TR, Bacci M, Bollazzi M (2015) Acromyrmex charruanus: a new inquiline social parasite species of leaf-cutting ants. Insectes Soc 62:335-349

Schär S, Nash DR (2014) Evidence that microgynes of Myrmica rubra ants are social parasites that attack old host colonies. J Evol Biol 27:2396-2407

Schrader L, Pan H, Bollazzi M, Schiøtt M, Larabee FJ, Bi X, Deng Y, Zhang G, Boomsma JJ, Rabeling C (2021) Relaxed selection underlies genome erosion in socially parasitic ant species. Nat Commun 12:1-3

Schultz TR, Bekkevold D, Boomsma JJ (1998) Acromyrmex insinuator new species: an incipient social parasite of fungus-growing ants. Insectes Soc 45:457-471

Sumner S, Hughes WOH, Boomsma JJ (2003a) Evidence for differential selection and potential adaptive evolution in the worker caste of an inquiline social parasite. Behav Ecol Sociobiol 54:256-263

Sumner S, Nash DR, Boomsma JJ (2003b) The adaptive significance of inquiline parasite workers. Proc Biol Sci 270:1315-1322

Sumner S, Nash DR, Boomsma JJ (2003c) The adaptive significance of inquiline parasite workers. Proc R Soc Lond Ser B Biol Sci 270:1315

Sumner S, Aanen DK, Delabie J, Boomsma JJ (2004) The evolution of social parasitism in Acromyrmex leaf-cutting ants: a test of emery's rule. Insectes Soc 51:37-42 